\title{
3M-CDNet: A Universal Powerful Benchmark for Remote Sensing Image Change Detection
}

\author{
Kaiqiang Song and Jie Jiang
}

\begin{abstract}
While deep learning-based methods have gained popularity and have made remarkable progress in remote sensing (RS) image change detection (CD), the limited amount of available data hinders the performance of most supervised methods. The CD networks transferred or derived from other fields can be fronted with a weak generalization capability. Developing a universal benchmark for performance evaluations based on the available datasets is urgent. To address these problems, we proposed a lightweight network, termed 3M-CDNet, which only requires about 3.12 $M$ parameters. The lighter the network, the easier it is to train and alleviate overfitting the limited amount of data, resulting in a better generalization capability. 3M-CDNet has a flexible modular design that achieves performance improvements by incorporating plug-and-play modules. 3MCDNet gains accuracy improvements in two ways: (1) the application of deformable convolutions (DConv) in the backbone network to gain a good geometric transformation modeling capacity for CD and (2) the application of an effective two-level feature fusion strategy to enhance the feature representation capacity. 3M-CDNet gains a good generalization capacity by incorporating effective "tricks" to alleviate overfitting, in which online data augmentation (Online $D A$ ) is applied to increase the diversity of the training samples, and Dropout regularization is applied in the classifier. Extensive ablation studies have proved the effectiveness of the core components. Experiment results suggest that 3M-CDNet outperforms state-of-the-art methods on several optical RS datasets and serves as a new universal benchmark. Specifically, 3M-CDNet achieves the best F1-score, i.e., LEVIRCD (0.9161), Season-Varying (0.9473), and DSIFN (0.7031).
\end{abstract}

Index Terms-Change detection (CD), convolutional neural networks (CNNs), deformable convolutions (DConv), optical remote sensing (RS) images.

\section{INTRODUCTION}

$\mathrm{C}$ HANGE detection (CD) aims to identify and locate the change footprints from multitemporal remote sensing (RS) images acquired over the same geographical region at different times. CD has a wide range of applications, such as land cover and land use identification, urbanization monitoring, and damage assessment [1]. With the rapid development of RS techniques, optical RS images are among the most representative data widely utilized for $\mathrm{CD}$. Especially the highand very high-resolution RS images, which can reflect abundant spectral and spatial information of geospatial objects, allow us to retain more details and obtain high-quality change maps. In this study, the main concern was binary CD based on optical RS images. CD methods usually generate a pixel-level change map, in which pixels are classified as changed or unchanged.

With the impressive breakthroughs made in deep learning,

The authors are with the School of Instrumentation and Optoelectronic Engineering, Key Laboratory of Precision Opto-mechatronics Technology, Ministry of Education, Beijing Advanced Innovation Center for Big DataBased Precision Medicine, Beihang University, Beijing 100191, China (e-mail: kaiqiangsong@buaa.edu.cn; jiangjie@buaa.edu.cn).
CD methods have gradually evolved from traditional [2] to deep neural network (DNN)-based methods [1]. Supervised CD methods built on convolutional neural networks (CNNs) have gained popularity and show promising performance. Some attempts adopted the U-shaped architecture [3]-[5], which concatenates feature maps from different levels through multiple skip connections to improve accuracy. These studies demonstrated that both high-level semantic information and low-level detail information are important in CD. Unfortunately, which multilevel feature fusion strategy is the better is not clear, and dense skip connections bring about heavy computational costs. Alternatively, recent works proposed attentionmechanism-based networks to learn discriminative features and alleviate distracting by pseudo-changes [6]-[10]. However, they usually require a large amount of data to achieve satisfactory performance; otherwise, it is easy to overfit.

Despite the increasing number of raw RS images, the timeconsuming and labor-intensive work of manual interpretation still hinders the development of $\mathrm{CD}$ methods considering the data-hungry nature of DNNs. Currently, only a few open available labelled datasets can be used for model training and evaluation, such as LEVIR-CD [6], Season-Varying [11], DSIFN [8], HRSCD [4]. Unfortunately, all of them have less data than the ImageNet and COCO datasets. While DNN-based $\mathrm{CD}$ methods have made remarkable progress, the limited data hinder the performance of most supervised methods. The CD networks derived from other CV tasks can be fronted with a weak generalization capability. Thus, overfitting becomes one of the main concerns. To address the problem, a lightweight CD model with few parameters becomes an intuitive solution.

Moreover, due to some works adopting different datasets for evaluation, it is difficult to say which one achieves the best performance. Although some methods are evaluated on the same dataset, unfortunately, they adopt different criteria to split the datasets [6], [7], which makes it difficult to compare them directly. In addition, only a few works are openly available to the public. The lack of implementation details results in poor reproducibility, which heavily hinders the development of $\mathrm{CD}$ algorithms from research to application. Therefore, developing a universal benchmark based on available datasets is urgent.

The main concerns of this study are as follows: (1) achieve a good generalization capability and (2) gain considerable accuracy improvements. Inspired by Occam's Razor ("Non sunt multiplicanda entia sine necessitate", or "Entities are not to be multiplied without necessity"), we propose a lightweight network called 3M-CDNet. To provide a universal benchmark with good reproducibility, all the implementation details will be

Color versions of one or more of the figures in this article are available online at http://ieeexplore.ieee.org. 


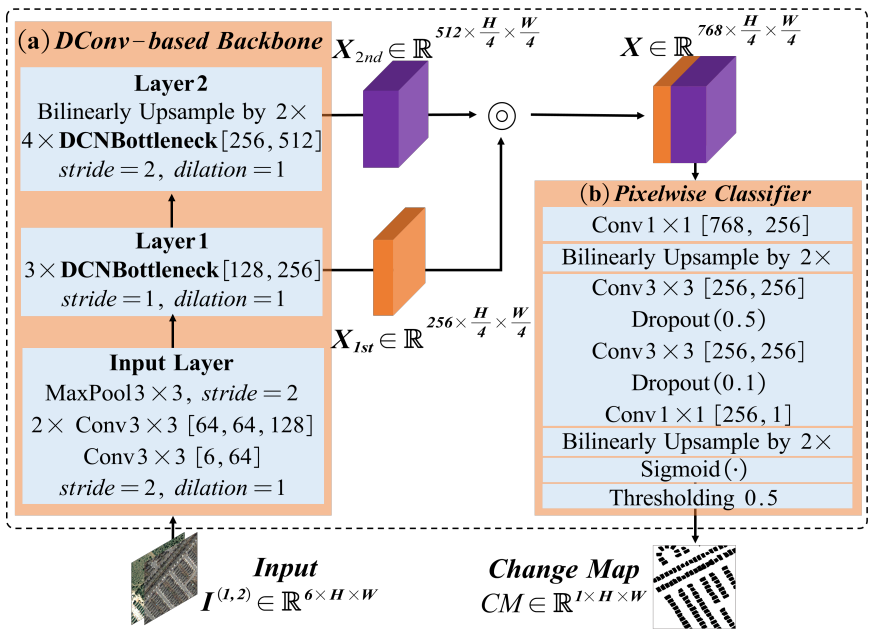

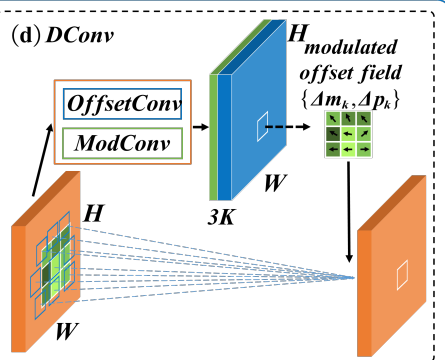

Number $\times$ Filter size $[$ in, mid, out $]$ channels Filter size $[$ in, out $]$ channels

Number $\times$ DCNBottleneck $[$ in, out $]$ channels concatenation along the channel axis elementwise sum

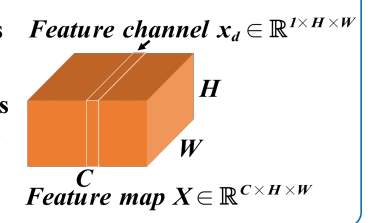

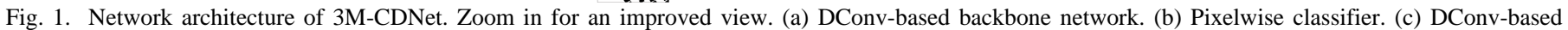
bottleneck residual blocks. (d) DConv. Best viewed in color. For brevity, ReLU and batch normalization (BN) at the tail of convolution layers are not fully shown. released at https://github.com/songkq/3M-CDNet. The main contributions are summarized as follows.

1) This paper presents a lightweight but powerful $3 \mathrm{M}-\mathrm{CDNet}$ as a universal benchmark for $\mathrm{CD}$ that only involves about $\mathbf{3 . 1 2}$ $\boldsymbol{M}$ parameters. $3 \mathrm{M}-\mathrm{CDNet}$ has a modular design with high flexibility for easy plug-in of plug-and-play modules, e.g., DConv [12], to gain performance improvements.

2) To the best of our knowledge, this study is among the first to incorporate DConv into the backbone network to enhance the geometric transformation modeling capacity for $\mathrm{CD}$.

3) This study explores a "bag of tricks" to achieve considerable performance improvements. Specifically, Online $D A$ and Dropout regularization [13] were used to improve the generalization capability. A two-level feature fusion strategy was applied to improve the feature representation capacity.

\section{PRoposed Method}

We proposed a universal benchmark for $\mathrm{CD}$, termed $3 \mathrm{M}$ CDNet, which only involves about $3.12 \mathrm{M}$ parameters. As shown in Fig. 1, 3M-CDNet mainly consists of two core components: (a) DConv-based backbone and (b) Pixelwise classifier. The former is used for feature extraction from input $I^{(1,2)} \in \mathbb{R}^{6 \times \boldsymbol{H} \times \boldsymbol{W}}$, e.g., taking as an input with 6 bands a pair of bitemporal RGB images. The latter is used to classify the extracted features into two classes, and then generate a binary change map $C M \in \mathbb{R}^{I \times \boldsymbol{H} \times \boldsymbol{W}}$, where pixels are either changed or unchanged. 3M-CDNet has a modular structure with high flexibility. It allows achieving performance improvement by incorporating some plug-and-play modules, such as DConv.

\section{A. Network Architecture}

1) DConv-based Backbone Network. As shown in Fig. 1(a), the backbone network of $3 \mathrm{M}$-CDNet is composed of the Input Layer, Layer 1, and Layer2 . The main concern is to reduce the size of input through consecutive downsampling and convolution operations, and extract features maps with varying degrees of semantics. Specifically, Input Layer consists of three stacked $3 \times 3$ convolutional layers followed by a MaxPool layer. Input Layer is applied to downsample the input by 4 times and transform $I^{(1,2)} \in \mathbb{R}^{6 \times \boldsymbol{H} \times \boldsymbol{W}}$ into a 3-D tensor $X_{0} \in \mathbb{R}^{128 \times \frac{H}{4} \times \frac{W}{4}}$, of which the spatial resolution is $1 / 4$ of the input size. $\boldsymbol{X}_{\text {Ist }} \in \mathbb{R}^{256 \times \frac{H}{4} \times \frac{W}{4}}$ and $\boldsymbol{X}_{2 n d} \in \mathbb{R}^{512 \times \frac{H}{4} \times \frac{W}{4}}$ are feature maps extracted from Layer 1 and Layer 2 , respectively.

(a) Introducing residual network [14]. When the $\mathrm{CNN}$ goes deeper, which could hamper the convergence, it may suffer from a degradation problem. Therefore, Layer 1 and Layer 2 adopt bottleneck residual blocks [14] as basic units, since residual blocks have the advantage of alleviating the degradation problem and promoting convergence during training. As shown in Fig. 1(c), bottleneck blocks can be formulated as follows:

$$
X_{\text {out }}^{l}=\operatorname{ReLU}\left(\boldsymbol{H}\left(X_{\text {in }}^{l}\right)+\zeta\left(X_{\text {in }}^{l}, \Omega_{l}\right)\right),
$$

where $X_{\text {in }}^{l}$ and $X_{\text {out }}^{l}$ are the input and output tensors of the $l$ th residual block, respectively. $\xi(\cdot)$ indicates the residual function, i.e., the right branch that consists of three stacked convolution layers. $H(\cdot)$ indicates the identity mapping function, i.e., the left branch. $H(\cdot)$ applies a downsampling projection shortcut through a Conv $1 \times 1 \_$BN layer only if stride is set to 2, e.g., the first block of Layer2, otherwise applying an identity shortcut. $\operatorname{Re} \boldsymbol{L} U(\cdot)$ is the rectified linear unit activation function for enhancing the non-linear fitting ability, and batch normalization $(\boldsymbol{B N})$ is also applied at the tail end of each convolution kernel to facilitate training procedure more stable.

(b) Introducing deformable convolutions [12]. Let $X_{\text {in }}(p)$ and $X_{\text {out }}(p)$ denote the feature at location $p$ of the input and output feature maps, respectively. Given a convolution kernel of $K$ sampling locations, let $w_{k}$ and $p_{k}$ denote the weight value and default offset for the $k$ th location of the kernel, respectively, e.g., a $3 \times 3$ kernel with $p_{k} \in\{(-1,-1),(-1,0), \ldots,(1,1) \mid K=9\}$. The deformable convolution [12] can be formulated as follows:

$$
X_{\text {out }}(p)=\sum_{k=1}^{K} w_{k} \cdot X_{\text {in }}\left(p+p_{k}+\Delta p_{k}\right) \cdot \Delta m_{k},
$$

where $\Delta p_{k}$ and $\Delta m_{k}$ are the learnable 2-D offsets and modulation factor for the $k$ th location, respectively, and $w_{k}$ enumerates the weights of the kernel. More precisely, two convolution layers OffsetConv and ModConv of the same kernel size are separately applied over the input feature maps to obtain $\Delta p_{k}$ and $\Delta m_{k}$. Due to the fractional coordinate 
$p+p_{k}+\Delta p_{k}$, the value of $X_{i n}\left(p+p_{k}+\Delta p_{k}\right)$ is calculated based on the values of the four surrounding integer points by bilinear interpolation. DConv consists of two steps: (1) generate deformable feature maps from the input feature maps based on the learned offsets in the $x$ and $y$ directions $\left\{\Delta p_{k}=\left(\Delta x_{k}, \Delta y_{k}\right)\right\}_{k=1}^{K}$ and apply the learned modulation factors $\left\{\Delta m_{k}\right\}_{k=1}^{K} \in(0,1)$ to modulate the activation of each location; and (2) apply a regular convolution over the deformable feature maps and then generate the output feature maps (see Fig. 1(d)).

DConvs are applied to replace all the $3 \times 3$ convolution layers of the bottleneck blocks in Layer 1 and Layer 2, termed DCNBottleneck (see Fig. 1(c)). In this way, the geometric transformation modeling capability of the backbone network can be enhanced. Therefore, 3M-CDNet has an advantage of addressing the pseudo-changes and overcoming the adverse effects of scale variations of objects with various shapes.

2) Pixelwise Classifier. The pixelwise classifier adopts a plain design with only four convolution layers. To obtain a change map of the same spatial resolution as the input, a 2-fold bilinear upsampling is applied after the first and last $1 \times 1$ convolution layers. The classifier classifies the extracted features by the backbone network into two classes and predicts a change probability map $C M_{\text {prob }} \in \mathbb{R}^{I \times \boldsymbol{H} \times \boldsymbol{W}}$ through a sigmoid layer, of which the values lie in the range $(0,1)$. Finally, the binary change map $C M \in \mathbb{R}^{I \times H \times W}$ can be generated by applying thresholding over $C M_{\text {prob }} \in \mathbb{R}^{1 \times \boldsymbol{H} \times W}$ with a threshold of 0.5 .

\section{B. Bag of Tricks for Performance Improvements}

We used a "bag of tricks" to gain considerable performance improvements. In particular, multilevel feature fusion serves as an indispensable role in improving accuracy of CD networks. Meanwhile, an intuitive solution to achieve good generalization capability comes from two aspects: (1) develop a lightweight model with few parameters and apply regularization; and (2) increase the diversity of the training samples.

1) Multilevel Feature Fusion Strategies. Previous works demonstrate that both high-level semantics and low-level detail information are important in CD. However, it is rare for these studies to clearly state which feature fusion strategy is effective. This work compares three kinds of feature fusion strategies as follows: (1) only apply the high-level feature maps $\boldsymbol{X}_{2 n d}$, termed the one-level strategy; (2) apply the fusion feature maps $\boldsymbol{X}$ obtained by concatenating the high-level $\boldsymbol{X}_{2 n d}$ and lowlevel $\boldsymbol{X}_{\text {Ist }}$ along the channel axis, i.e., $\boldsymbol{X}=\boldsymbol{X}_{1 s t} \odot \boldsymbol{X}_{2 n d}$, termed the two-level strategy; and (3) apply the two-level strategy and then apply an extra fusion feature map $\hat{\boldsymbol{X}} \in \mathbb{R}^{384 \times \frac{H}{4} \times \frac{W}{4}}$, which is obtained by concatenating the output feature maps of the first $1 \times 1$ convolution layer of the classifier and $\boldsymbol{X}_{\boldsymbol{\theta}}$ extracted by Input Layer, termed the three-level strategy. Although some channel attention modules, e.g., CBAM [15], can be applied for improvements, the concatenation operation was selected for its simplicity to achieve high computational efficiency using the minimal number of parameters.

2) Dropout Regularization [13]. Dropout is a simple yet effective way to prevent neural networks from overfitting.
During training, Dropout randomly drops units from the network with a certain probability $p_{d}$, which can be equivalent to training numerous different networks simultaneously, i.e., $D(X)=D_{m} \odot X$, where $D_{m}$ indicates a binary mask of the same size with the feature map $X$, and $\odot$ indicates the element-wise multiplication operation. $D_{m}$ is randomly generated from a Bernoulli distribution with a probability $p_{d}$, and the units of the feature maps corresponding to the locations of zeros are to be discarded during training. At test time, a neural unit is always presented, and the weights are multiplied by $p_{d}$ so that the output of the unit would be the same as the expected output at training time, i.e., $D(X)=p_{d} \cdot X$. As shown in Fig. 1(b), two Dropout layers with probabilities of 0.5 and 0.1 are applied at the tail end of the classifier's $3 \times 3$ convolution layers.

3) Online Data Augmentation. Data augmentation $(\boldsymbol{D A})$ is a simple yet effective technique for regularizing the network. $\boldsymbol{D A}$ can be used to simulate scale variations, illumination variations, and pseudo-changes, such as the spectra changes between bitemporal images. Online $\boldsymbol{D A}$ means that do $\boldsymbol{D A}$ only when training instead of expanding the training set at the cost of expensive training time. Online $\boldsymbol{D A}$ was randomly applied over every batch data with a probability of 0.8 by randomly shiftingrotating-scaling with padding zeros, rotating by $90^{\circ}, 180^{\circ}$, and $270^{\circ}$, and flipping in horizontal and vertical directions, and applying color jitter. Each kind of augmentation was randomly applied with a probability of 0.5 .

\section{Loss Function Definition}

During training, network parameters are iteratively updated by minimizing the loss between the forward output of $3 \mathrm{M}$ $\mathrm{CDNet}$ and the reference change map with the $\boldsymbol{B P}$ algorithm according to a specific loss function. Since CD aims to classify the pixels as changed or unchanged, binary cross-entropy $(B C E)$ loss is a natural candidate. Due to the widespread class imbalance, dominant unchanged pixels would make models tend to collapse. Thus, a soft Jaccard term is introduced, and the loss function can be formulated as shown in Equation (3).

$$
\begin{aligned}
\boldsymbol{L}_{B C D}= & -\lambda \frac{1}{N} \sum_{n=1}^{N}\left(y_{n} \log \left(\hat{y}_{n}\right)+\left(1-y_{n}\right) \log \left(1-\hat{y}_{n}\right)\right) \\
& +(1-\lambda) \frac{1}{N} \log \sum_{n=1}^{N} \frac{y_{n} \cdot \hat{y}_{n}}{y_{n}+\hat{y}_{n}-y_{n} \cdot \hat{y}_{n}}
\end{aligned}
$$

where two terms indicate the $B C E$ loss and soft Jaccard with the weights $\lambda$ and $1-\lambda$ respectively, and $\lambda$ is empirically set to 0.7. $N$ is the number of training samples, and $\hat{y}_{n}$ and $y_{n}$ are pixels of predicted change maps and the reference, respectively.

\section{EXPERIMENTS}

\section{A. Evaluation Metrics and Experiment Settings}

The most common metrics related to the changed category were adopted for the quantitative evaluation, including overall accuracy $(\boldsymbol{O A})$, precision $(\boldsymbol{P r})$, recall $(\boldsymbol{R e})$, F1-score $\left(\boldsymbol{F}_{\boldsymbol{I}}\right)$, and the intersection of union $(\boldsymbol{I o U}) . \boldsymbol{F}_{\boldsymbol{I}}$ and $\boldsymbol{I o} \boldsymbol{U}$ are comprehensive indicators; the higher the value, the better the performance.

The proposed 3M-CDNet was implemented using PyTorch framework and optimized by minimizing Eq. (3) through the 
TABLE II

Results Of ABlation StUdies ON THE LEVIR-CD, SEASON-VARYing, AND DSIFN DATASET

\begin{tabular}{|c|c|c|c|c|c|c|c|c|c|}
\hline \multirow{2}{*}{ Method } & \multicolumn{3}{|c|}{ LEVIR-CD } & \multicolumn{3}{|c|}{ Season-Varying } & \multicolumn{3}{|c|}{ DSIFN } \\
\hline & $O A(\%)$ & IoU & $F_{1}$ & $O A(\%)$ & IoU & $F_{1}$ & $O A(\%)$ & $I o U$ & $F_{1}$ \\
\hline w/ two-level & $99.15^{*}$ & $0.8452 *$ & $0.9161^{*}$ & 98.62 & 0.8995 & 0.9471 & 89.21 & $0.5422 *$ & $0.7031 *$ \\
\hline w/ three-level & 99.05 & 0.8291 & 0.9066 & 98.48 & 0.8899 & 0.9417 & 85.80 & 0.4783 & 0.6471 \\
\hline w/ one-level & 99.03 & 0.8243 & 0.9037 & 98.19 & 0.8720 & 0.9316 & 88.06 & 0.4926 & 0.6600 \\
\hline w/o DA & 99.01 & 0.8212 & 0.9018 & 98.64* & $0.8998^{*}$ & $0.9473^{*}$ & $89.72 *$ & 0.5128 & 0.6779 \\
\hline w/o DA/Dropout & 98.95 & 0.8109 & 0.8956 & 98.47 & 0.8892 & 0.9414 & 88.00 & 0.4879 & 0.6558 \\
\hline w/o DA/DConv & 98.92 & 0.8069 & 0.8932 & 98.25 & 0.8731 & 0.9323 & 88.45 & 0.4683 & 0.6379 \\
\hline w/o DConv & 99.04 & 0.8283 & 0.9061 & 98.06 & 0.8635 & 0.9268 & 89.07 & 0.5125 & 0.6777 \\
\hline
\end{tabular}

AdamW optimizer [16] with $\beta_{1}=0.9$ and $\beta_{2}=0.99$, of which the initial learning rate and weight decay were set to 0.000125 and 0.0005 , respectively. The minibatch size was set to 16 on an NVIDIA RTX 3090 GPU with 24 GB memory. For evaluation, we adopted three representative datasets, i.e., LEVIR-CD [6], Season-Varying [11], and DSIFN [8]; we applied the criteria as recommended by the creators to split the datasets (see Table I).

TABLE I

EXPERIMENTAL DATASETS

\begin{tabular}{lrrrr}
\hline \multirow{2}{*}{ Datasets } & Size of & \multicolumn{3}{c}{ Number of Samples } \\
\cline { 3 - 5 } & Samples & training & validation & test \\
\hline LEVIR-CD & $512 \times 512$ & 4,016 & 1,024 & 512 \\
Season-Varying & $256 \times 256$ & 10,000 & 3,000 & 3,000 \\
DSIFN & $512 \times 512$ & 3,546 & 394 & 48 \\
\hline
\end{tabular}

B. Ablation Studies

Ablation studies have been conducted to verify each component's contribution of 3M-CDNet. Table II presents the quantitative results on several public datasets, where "w/o" and "w/" mean "without" and "with", respectively.

1) Effects of Multilevel Feature Fusion Strategies. The first three rows of the Method applied with all components were used to verify the contributions of three different kinds of multilevel feature fusion strategies. Table II suggests that the two-level strategy always achieves the best performance in terms of $\boldsymbol{F}_{\boldsymbol{l}}$, i.e., LEVIR-CD (0.9161), Season-Varying (0.9471), and DSIFN (0.7031). Compared with the one-level strategy that lacks low-level details, it increased $\boldsymbol{I o U}$ and $\boldsymbol{F}_{\boldsymbol{I}}$ significantly by about $2.09 \%$ and $1.24 \%$ on LEVIR-CD, $2.75 \%$ and $1.55 \%$ on Season-Varying, as well as $4.96 \%$ and $4.31 \%$ on DSIFN, respectively. Unfortunately, the impact of the threelevel strategy is negligible when using samples with size $512 \times 512$ for model training, e.g., as shown in the LEVIR-CD and DSIFN. However, the three-level strategy helps to achieve an improvement of $\boldsymbol{F}_{\boldsymbol{I}}(1.01 \%)$ and $\boldsymbol{I o U}(1.79 \%)$, compared to the one-level strategy, because it provides more spatial details in the case of using samples with the smaller size of $256 \times 256$ on Season-Varying. We can conclude that the two-level strategy is enough for improvements in our case, while introducing either insufficient or excessive features could bring about an unexpected degradation problem.

2) Effects of Different Tricks. The last four rows of the Method based on the two-level strategy were used to verify the effectiveness of Online DA, Dropout, and DConv. Table II suggests that Online $\boldsymbol{D A}$ makes the most impressive contributions on the smaller datasets, i.e., LEVIR-CD and DSIFN. Compared with the situation without $\boldsymbol{D A}$, applying $\boldsymbol{D A}$ achieves considerable improvements of $\boldsymbol{F}_{\boldsymbol{1}}(1.43 \%)$ and $\boldsymbol{I o} \boldsymbol{U}$ $(2.40 \%)$ on LEVIR-CD. Similar situations can be observed on
DSIFN. It demonstrates that Online $\boldsymbol{D A}$ is an effective trick to achieve immediate gains through improving the diversity of samples, especially when lacking enough training samples. Otherwise, we can observe that its impact is negligible in the case of Season-Varying dataset with sufficient samples. Meanwhile, Dropout can be an effective complementary regularization with Online $\boldsymbol{D A}$ for achieving good generalization capacity. In addition, Table II shows that DConv serves as an indispensable component for achieving high accuracy. For an instance, the last row shows that performance drops significantly without $\boldsymbol{D C} \boldsymbol{C o n v}$, where $\boldsymbol{F}_{\boldsymbol{1}}$ decreased by about $1 \%, 2.03 \%$, and $2.54 \%$ on the three datasets, and $\mathbf{I o U}$ decreased by $1.69 \%, 3.60 \%$, and $2.97 \%$, respectively. In particular, DConv helps to improve the generalization capacities in the challenging case of DSIFN, in which training and testing samples are collected from different cities.

\section{Comparisons with Other Approaches}

1) Comparisons of Network Parameters and Computational Cost. Table III shows the computational costs (GFLOPs) and number of parameters $(\boldsymbol{M})$ of the CD networks. When calculating the computational cost during testing, it takes as an input $6 \times 256 \times 256$ and $6 \times 512 \times 512$ fixed-size. $3 \mathrm{M}-\mathrm{CDNet}$ costs $23.66 \boldsymbol{G}$ and $94.64 \boldsymbol{G F L O P s}$ according to the input sizes.

2) Comparisons on LEVIR-CD. W-Net [3], FC-EF-Res [4], and Peng et al.[5], and attention-based methods STANet [6], DDCNN [7], and FarSeg [17] were selected as benchmarks. In particular, STANet was proposed by the dataset's creator. Table IV presents the quantitative results and suggests that $3 \mathrm{M}$ CDNet outperforms the state-of-the-art FarSeg/DDCNN (w/ Online $\boldsymbol{D A}$ ) and achieves the best performance in terms of $\mathbf{I o U}$ $(0.8452)$ and $\boldsymbol{F}_{\boldsymbol{I}}(0.9161)$, which increased by about $1.66 \%$ and $0.98 \%$ compared to FarSeg, respectively. Moreover, 3MCDNet makes a better trade-off between precision and recall than other approaches. 3M-CDNet has broken the record and become the new benchmark for the LEVIR-CD dataset. For intuitive comparisons, the visual results are shown in Fig. 2. Compared with the change maps generated by other methods, 3M-CDNet achieves more complete and accurate boundaries and higher internal compactness on objects with various scales and shapes, which is more consistent with the reference. Moreover, 3M-CDNet successfully identifies the tiny gap among the crowded building groups and overcomes the pseudochanges caused by the spectra changes. However, other methods suffer from more false alarms.

3) Comparisons on Season-Varying. More attention-based methods IFN [8], BA ${ }^{2}$ Net [9], and DASNet [10] were selected as benchmarks for the quantitative evaluation. Table $\mathrm{V}$ suggests that $3 \mathrm{M}-\mathrm{CDNet}$ is consistently superior to other approaches in 
TABLE III

\begin{tabular}{|c|c|c|c|}
\hline \multirow[t]{2}{*}{ Method } & \multirow{2}{*}{$\begin{array}{c}\text { Number of } \\
\text { Parameters } \\
\text { (M) }\end{array}$} & \multicolumn{2}{|c|}{$\begin{array}{c}\text { Computational Costs } \\
(\text { GFLOPs })\end{array}$} \\
\hline & & $512 \times 512$ & $256 \times 256$ \\
\hline DDCNN [6] & 60.21 & 855.59 & 213.90 \\
\hline IFN [7] & 35.99 & 328.87 & 82.22 \\
\hline W-Net [2] & 42.55 & 290.82 & 72.70 \\
\hline STANet [5] & 16.93 & 155.33 & 19.58 \\
\hline Peng et al. [4] & 9.04 & 125.35 & 31.34 \\
\hline FarSeg [16] & 31.38 & 41.74 & 11.94 \\
\hline FC-EF-Res [3] & 1.10 & 6.89 & 1.72 \\
\hline 3M-CDNet & 3.12 & 94.64 & 23.66 \\
\hline
\end{tabular}

TABLE IV

COMPARISON OF RESULTS ON THE LEVIR-CD DATASET

\begin{tabular}{lllll}
\hline Method & $\boldsymbol{P r}(\mathbf{\%})$ & $\boldsymbol{R e}(\mathbf{\%})$ & $\boldsymbol{I o U}$ & $\boldsymbol{F}_{\boldsymbol{I}}$ \\
\hline STANet [5] & 85.01 & $91.38^{*}$ & 0.7869 & 0.8808 \\
W-Net [2] & 90.37 & 85.92 & 0.7871 & 0.8809 \\
FC-EF-Res [3] & 90.22 & 88.25 & 0.8055 & 0.8923 \\
Peng et al. [4] & 91.53 & 88.70 & 0.8197 & 0.9009 \\
DDCNN [6] & $92.15^{*}$ & 89.07 & 0.8279 & 0.9059 \\
FarSeg [16] & 91.04 & 90.22 & 0.8286 & 0.9063 \\
3M-CDNet & 91.99 & 91.24 & $0.8452^{*}$ & $0.9161^{*}$ \\
\hline
\end{tabular}

TABLE V

COMPARISON OF RESULTS ON THE SEASON-VARYING DATASET

\begin{tabular}{lllll}
\hline Method & $\boldsymbol{O A}(\mathbf{\%})$ & $\boldsymbol{P r}(\%)$ & $\boldsymbol{R e}(\mathbf{\%})$ & $\boldsymbol{F}_{\boldsymbol{1}}$ \\
\hline Peng et al. [4] & 96.73 & 89.54 & 87.11 & 0.8756 \\
FC-EF-Res [3] & 97.25 & 89.91 & 87.37 & 0.8862 \\
IFN [7] & 97.71 & 94.96 & 86.08 & 0.9030 \\
BA $^{2}$ Net [8] & $98.94 *$ & 88.12 & 95.28 & 0.9136 \\
DASNet [9] & 98.20 & 92.20 & 93.20 & 0.9270 \\
FarSeg [16] & 98.23 & 87.75 & $99.56^{*}$ & 0.9328 \\
DDCNN [6] & 98.64 & $96.71 *$ & 92.32 & 0.9446 \\
3M-CDNet & 98.64 & 90.78 & 99.04 & $0.9473 *$ \\
\hline
\end{tabular}

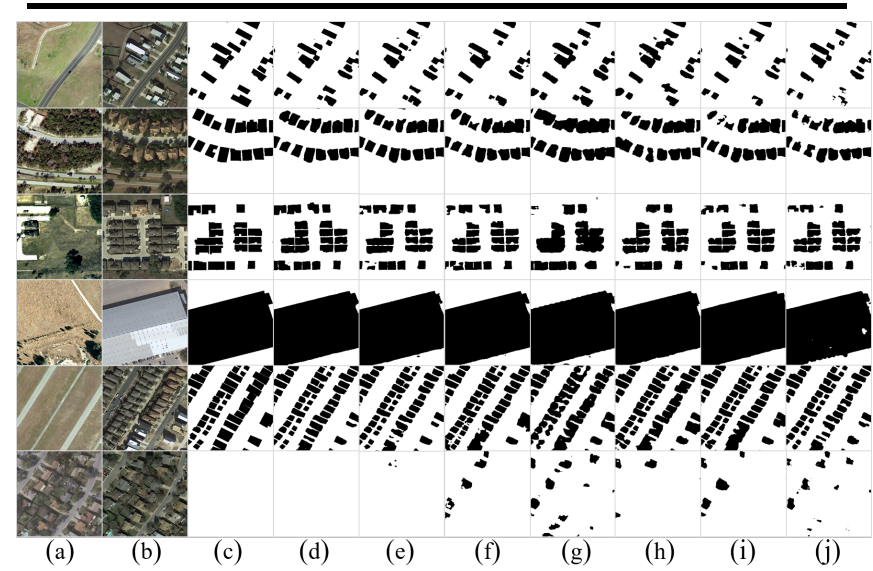

Fig. 2. Comparisons on the LEVIR-CD dataset. Zoom in for an improved view. (a) Image T1. (b) Image T2. (c) Reference change map. (d) 3M-CDNet. (e) FarSeg. (f) DDCNN. (g) STANet. (h) FC-EF-Res. (i) Peng et al. (j) W-Net.

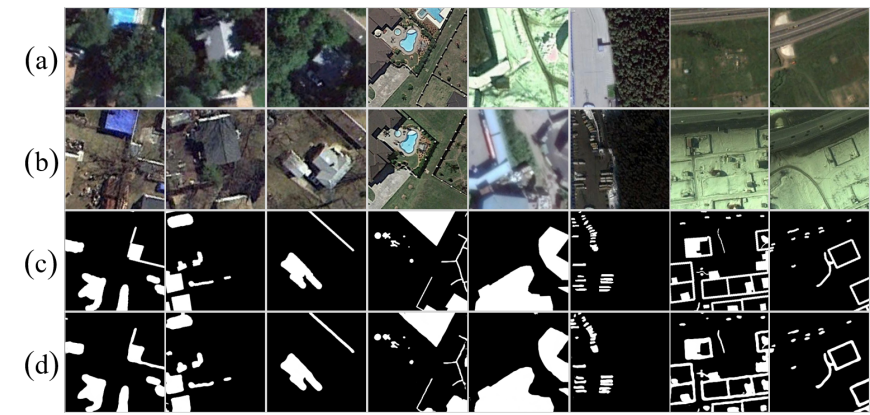

Fig. 3. CD results on the Season-Varying dataset. Zoom in for an improved view. (a) Image T1. (b) Image T2. (c) Reference change map. (d) 3M-CDNet. terms of $\boldsymbol{F}_{\boldsymbol{I}}(0.9473)$. Although DDCNN achieves very similar results to $3 \mathrm{M}-\mathrm{CDNet}$, 3M-CDNet requires fewer parameters than DDCNN and remarkably reduces the computational cost. Table III shows the number of parameters of DDCNN (60.21 $\boldsymbol{M})$ is about 19 times that of 3M-CDNet (3.12 M), and it has dense skip connections that bring about 213.90 GFLOPs computational costs, i.e., about 9 times of 3M-CDNet. Fig. 3 shows even in the challenging case of large seasonal variations (summer-to-winter/autumn), 3M-CDNet accurately identifies the changed objects of various scales and appearance, including the road changes and building changes.

\section{CONCLUSIONS}

The universal network $3 \mathrm{M}-\mathrm{CDNet}$ is proposed for CD. The lightweight 3M-CDNet exhibits a good generalization capacity by incorporating effective tricks, i.e., Online DA and Dropout regularization. The flexible $3 \mathrm{M}-\mathrm{CDNet}$ achieves accuracy improvements in two ways. One is by applying DConv in the backbone network to gain good geometric transformation modeling capacity for objects with various scales and appearance. Another is by applying a two-level feature fusion strategy to enhance the feature representation capacity. Experiment results show 3M-CDNet broke the record for accuracy compared to the state-of-the-art methods and is the new benchmark on three public datasets.

\section{REFERENCES}

[1] W. Shi, Z. Min, R. Zhang, S. Chen, and Z. Zhan, "Change detection based on artificial intelligence: State-of-the-art and challenges," Remote Sensing, vol. 12, pp. 1688, May 2020.

[2] T. Celik, "Unsupervised change detection in satellite images using principal component analysis and k-Means clustering," IEEE Geosci. Remote Sens. Lett., vol. 6 , no. 4 , pp. $772-776$, Oct. 2009

[3] B. Hou, Q. Liu, H. Wang, and Y. Wang, "From W-Net to CDGAN: Bitemporal change detection via deep learning techniques," IEEE Trans. Geosci. Remote Sens., vol. 58, no. 3, pp. 1790-1802, Mar. 2020.

[4] R. C. Daudt, B. Le Saux, A. Boulch, and Y. Gousseau, "Multitask learning for largescale semantic change detection," Comput. Vision Image Understanding, vol. 187, pp. 102783 , Oct. 2019.

[5] D. Peng, Y. Zhang, and H. Guan, "End-to-end change detection for high resolution satellite images using improved UNET++," Remote Sensing, vol. 11, no. 11, pp. 1382, Jun. 2019.

[6] H. Chen and Z. Shi, "A spatial-temporal attention-based method and a new dataset for remote sensing image change detection," Remote Sensing, vol. 12, no. 10, pp. 1662, May 2020.

[7] X. Peng, R. Zhong, Z. Li, and Q. Li, "Optical remote sensing image change detection based on attention mechanism and image difference," IEEE Trans. Geosci. Remote Sens., pp. 1-12, 2020.

[8] C. Zhang, P. Yue, D. Tapete, L. Jiang, B. Shangguan, L. Huang, and G. Liu, "A deeply supervised image fusion network for change detection in high resolution bitemporal remote sensing images," ISPRS J. Photogramm. Remote Sens., vol. 166, pp. 183-200, Aug. 2020

[9] Y. Zhang, S. Zhang, Y. Li, and Y. Zhang, "Coarse-to-fine satellite images change detection framework via boundary-aware attentive network," Sensors, vol. 20, no. 23, pp. 6735, Nov. 2020.

[10] J. Chen, Z. Yuan, J. Peng, L. Chen, H. Haozhe, J. Zhu, Y. Liu, and H. Li, "DASNet: Dual attentive fully convolutional siamese networks for change detection of high resolution satellite images," IEEE J. Sel. Top. Appl. Earth Obs. Remote Sens., pp. 11, Nov. 2020.

[11] M. Lebedev, Y. V. Vizilter, O. Vygolov, V. Knyaz, and A. Y. Rubis, "Change detection in remote sensing images using conditional adversarial networks," International Archives of the Photogrammetry, Remote Sensing \& Spatial Information Sciences, vol. 42, no. 2, pp. 565-571, Jun. 2018.

[12] X. Zhu, H. Hu, S. Lin, and J. Dai, "Deformable ConvNets v2: More deformable, better results," in Proc. CVPR, LGB, CA, USA, Jun. 2019, pp. 9300-9308.

[13] N. Srivastava, G. Hinton, A. Krizhevsky, I. Sutskever, and R. Salakhutdinov, "Dropout: A simple way to prevent neural networks from overfitting," J. Mach. Learn. Res., vol. 15, no. 1, pp. 1929-1958, 2014.

[14] K. He, X. Zhang, S. Ren, and J. Sun, "Deep residual learning for image recognition," in Proc. CVPR, LAS, NV, USA, Jun. 2016, pp. 770-778.

[15] S. Woo, J. Park, J.-Y. Lee, and I. So Kweon, "CBAM: Convolutional block attention module," in Proc. ECCV, Munich, Germany, Sep. 2018, pp. 3-19.

[16] I. Loshchilov and F. Hutter, "Decoupled weight decay regularization," in Proc. ICLR, NO, LA, USA, May 2019.

[17] Z. Zheng, Y. Zhong, J. Wang, and A. Ma, "Foreground-aware relation network for geospatial object segmentation in high spatial resolution remote sensing imagery," in Proc. CVPR, SEA, WA, USA, Jun. 2020, pp. 4095-4104. 